\title{
La diversité des abeilles parisiennes
}

\author{
par Lise ROPARS***, Isabelle DAJOZ** et Benoît GESLIN*
}

\begin{abstract}
Résumé. Malgré les perturbations dues à l'urbanisation grandissante, les villes abritent une biodiversité non négligeable. Comme beaucoup d'autres taxons, les abeilles sauvages peuvent être relativement abondantes dans les zones urbaines du fait de certaines conditions favorables par rapport aux milieux agricoles (absence de pesticides, îlot de chaleur urbain, floraison toute l'année). Dans cette étude, nous compilons nos travaux conjoints afin d'enrichir la liste des espèces d'abeilles parisiennes. Au total, le suivi de 4 années (2011 puis 2014, 2015, 2016) a permis de recenser 87 espèces d'abeilles à Paris intra-muros. L'ajout en 2016 de la méthode de capture avec des filets à papillon en plus de la méthode passive avec coupelles colorées a permis de capturer 11 espèces supplémentaires. L'assemblage, qui représente à ce jour $8,8 \%$ de la diversité spécifique des abeilles sauvages de France métropolitaine, est cependant dominé par les Halictidae et ne compte que quelques espèces cleptoparasites, témoignant d'une communauté appauvrie.
\end{abstract}

\begin{abstract}
Despite the forever increasing urbanization, cities often shelter a significant biodiversity. As many other taxa, wild bees may find refuge in urban areas because of relative favorable conditions compared to intensive agricultural environments. In this study, we compiled our joint work to extend the list of Parisian bees. Over 4 years of monitoring $(2011,2014,2015,2016)$, we recorded 87 bee species in intra-muros Paris. In 2016 we added net captures to our sampling procedures with colored pan traps, which provided 11 additional species. The assemblage now represents $8.8 \%$ of the French fauna of Apoïdae. However, this assemblage is mostly dominated by Halictidae and includes only few kleptoparasitic species highlighting an impoverished community.
\end{abstract}

Mots-clés : abeilles sauvages, Paris, écologie urbaine.

Keywords : wild bees, Paris, urban ecology.

\section{Introduction}

Les insectes pollinisateurs sont en fort déclin depuis une soixantaine d'années (POTTs et al. 2016). Plusieurs facteurs sont à l'origine de cette régression notamment la destruction des habitats naturels, l'utilisation de pesticides, la perte de ressources florales ou encore l'introduction d'espèces envahissantes (GOULSON et al. 2015). En dépit de l'effet négatif de l'urbanisation sur les communautés de pollinisateurs (DEGUINES et al. 2016), plusieurs études ont montré que les villes pouvaient abriter un certain nombre d'espèces d'abeilles (BALDOCK et al. 2015; HALL et al. 2016). Par exemple, 291 espèces ont été recensées sur le Grand Lyon sur 2 ans d'échantillonnage (FORTEL

\footnotetext{
${ }^{*}$ Aix Marseille Univ, Univ Avignon, CNRS, IRD, IMBE, Marseille, France

** Institut d'Ecologie et des Sciences de l'Environnement de Paris (iEES Paris UMR CNRS 76/8), Equipe Ecologie et Evolution des réseaux d'interactions, Université Paris Diderot, CNRS-UPMC.
}

et al. 2014). Les habitats favorables pour les abeilles sauvages doivent à la fois proposer une offre de nourriture suffisante ainsi que des sites de nidifications variés (WESTRICH 1996). Contrairement à une grande partie des espaces agricoles actuels, les espaces verts présents en zones urbaines et péri-urbaines offrent une grande diversité d'espèces de plantes à fleurs, sont moins traités avec des pesticides et bénéficient de températures en moyennes plus douces dues à l'îlot de chaleur urbain (BALDOCK et al. 2015).

En 2011, GESLIN et al. (2016) ont dénombré 44 espèces d'abeilles dans les parcs urbains de Paris. En 2014 et 2015, nos travaux conjoints ont permis d'étendre cette liste à 67 espèces (ROPARS et al. 2017). Nous présentons ici une mise à jour des espèces présentes à Paris avec l'ajout des spécimens capturés en 2016. 


\section{Matériel et Méthodes}

\section{Sites d'études}

Toutes les captures d'abeilles ont été conduites dans la ville de Paris, qui couvre près de $105 \mathrm{~km}^{2}$. Cette ville possède plus de 2.2 millions d'habitants (INSEE 2014) et dispose seulement de 3000 ha d'espaces verts ce qui équivaut à $14,5 \mathrm{~m}^{2}$ ou $5,8 \mathrm{~m}^{2}$ de surface végétalisée par habitant si on prend en compte ou non la surface des deux bois urbains (Vincennes et Boulogne) (APUR 2004). Cela est faible par rapport aux capitales voisines avec $36 \mathrm{~m}^{2}$ d'espaces verts par habitant à Amsterdam, $45 \mathrm{~m}^{2}$ à Londres, $59 \mathrm{~m}^{2}$ à Bruxelles ou encore $321 \mathrm{~m}^{2}$ à Rome (APUR 2004). Le climat parisien est océanique avec une température moyenne annuelle de $12,4^{\circ} \mathrm{C}$, un minimum de $4,9^{\circ} \mathrm{C}$ en janvier et un maximum de $20,5^{\circ} \mathrm{C}$ en juillet (http://www.infoclimat.fr).

\section{Suivi des abeilles sauvages}

Dans cette étude, nous compilons les données des espèces capturées en 2011, 2014, 2015 et 2016. Les sites sont localisés dans des squares, parcs urbains ou campus universitaires de Paris (figure 1). Pour chacun d'entre eux, les abeilles ont été capturées soit par méthode active soit par méthode passive soit en employant l'une et l'autre et systématiquement lorsque les conditions météorologiques étaient favorables (température $>12^{\circ} \mathrm{C}$, journée ensoleillée et faible vent). La méthode a consisté à capturer au filet pendant 15 minutes toutes les abeilles en train de butiner sur des patchs de végétation très fleuris $\left(3 \mathrm{~m}^{2}\right)$. La méthode passive, quant à elle, a consisté en l'emploi de pièges sous la forme de coupelles colorés (une blanche, une jaune, une bleue) laissés sur le terrain $24 \mathrm{~h}$ et préalablement peintes avec des peintures reflétant les ultra-violets auxquels sont sensibles les abeilles. Pour rendre effectif le piégeage, chaque coupelle doit être remplie d'eau (légèrement) savonneuse. Sur une station, les trois coupelles sont disposées linéairement et espacées d'1 m entre elles (WeStPHAL et al. 2008). Ces deux méthodes se complètent puisque celle dite passive sous-estime la présence et l'abondance des abeilles les plus grandes (Bombus, Colletes) tandis que la méthode active sous-estime la présence des abeilles les plus petites (Halictus, Lasioglossum) (WILSON et al. 2008 ; ROULSTON et al. 2007).
Le détail du protocole a varié d'une année à l'autre. En voici les détails :

- en 2011, GESLIN et al. (2016) ont capturé par méthode passive pendant 15 sessions d'échantillonnage de mi-avril à mi-juillet dans 3 grands parcs parisiens à savoir le Jardin des Plantes, le Jardin du Luxembourg et le Jardin de la Cité Internationale Universitaire de Paris ; - en 2014, Ropars et al. (2017) ont capturé par méthode passive pendant 10 sessions d'échantillonnage de mi-mai à mi-juillet dans 5 localités qui sont les campus des Universités Pierre et Marie Curie, Paris Descartes et Paris Diderot, de l'Institut de Physique du Globe de Paris et le Parc de Bercy (un essai expérimental a également été conduit à la Bibliothèque Nationale de France avec une seule pose de coupelles colorées - ayant fourni 4 abeilles) ;

- en 2015 , les 5 localités précédentes ont été rééchantillonnées par méthode passive avec l'ajout de 2 nouvelles localités (Jardin du Collège de France et Jardin de la Bibliothèque Nationale de France) pendant 13 sessions d'échantillonnage de mi-avril à mi-juillet.

- en 2016, les 7 localités précédentes ont été inventoriées pour la $3^{\mathrm{e}}$ année consécutive employant systématiquement les deux méthodes pendant 12 sessions d'échantillonnage de début avril à mi-juillet.

Les spécimens capturés dans les coupelles ont été conservés dans de l'alcool à $70^{\circ}$ jusqu'à leur préparation (rinçage, épinglage, séchage) dans le but de les déterminer à l'espèce. Ceux capturés au filet ont été tués en les plaçant dans des bocaux avec des granulés imbibés d'acétate d'éthyle. Ils ont ensuite été épinglés et déterminés suivant la nomenclature de KUHLMANN et al. (2013).

Enfin, à partir des données de la littérature, les informations sur les traits écologiques ont été renseignées pour chaque espèce. Les traits écologiques qui ont été considérés sont le type de nidification, la sociabilité, le caractère parasite et le lectisme (GENOUD 2017 ; Atlas Hymenoptera ; FlorAbeilles) ainsi que le statut de conservation tel que défini dans la liste rouge Européenne (NIETO et al. 2014). 


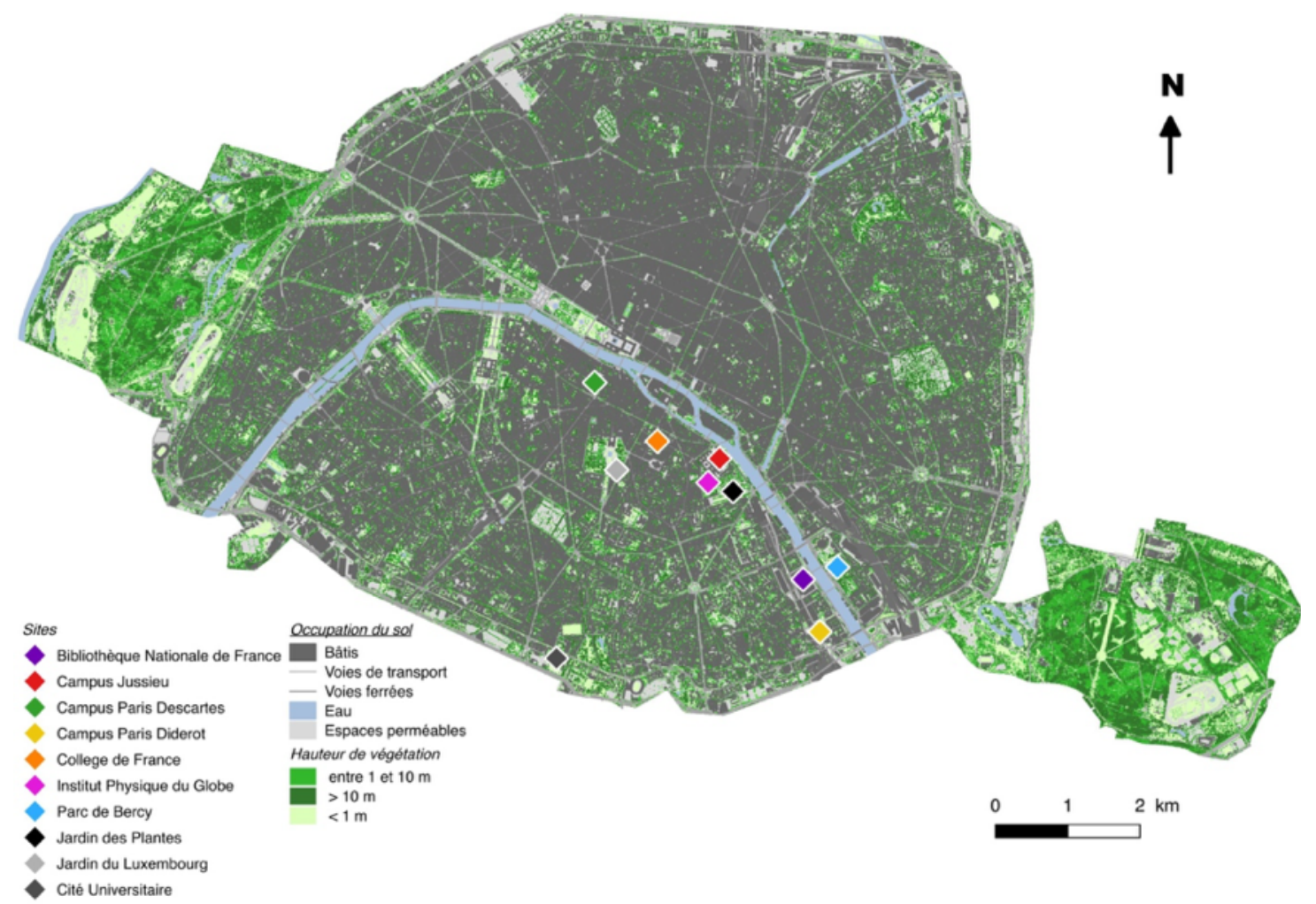

Figure $n^{\circ} \mathrm{I}$. Cartographie des sites échantillonnés. (en noir et gris, les sites de l'étude de Geslin et al. 2016 ; en couleurs, ceux de Ropars et al. 2017)

\section{Résultats}

\section{Caractérisation des abeilles parisiennes}

$\mathrm{Au}$ cours de l'année 2016, un total de 292 individus a été récolté dont 131 par piégeage passif et 161 par méthode active. Ils représentent 6 familles (Andrenidae, Apidae, Colletidae, Halictidae, Megachilidae, Melittidae), 16 genres et 55 espèces (la liste est disponible ici). A ce jour, seuls 22 spécimens du genre Hylaeus restent à déterminer. Les Halictidae dominent l'échantillon avec $33,2 \%$ de l'abondance totale suivis ensuite par les Apidae avec 29,4\% et par les Megachilidae avec 21,5\%. Les Andrènes constituent le genre le plus diversement représenté avec 23 espèces alors que seule une espèce de Sphecodes a été inventoriée. L'espèce la plus fréquemment rencontrée est Lasioglossum morio avec 31 individus soit 10,6\% du total des abeilles capturées en 2016. Elle est suivie par Anthophora plumipes avec 27 individus $(9,2 \%$ du total) et par Bombus pascuorum et Lasioglossum nitidulum avec 23 individus chacun $(7,8 \%$ du total des captures dans les deux cas). La plupart des espèces ne sont représentées que par quelques individus : 43 espèces le sont par moins de 5 individus dont 23 seulement par un seul individu. Le détail de la richesse spécifique mise en évidence par année est le suivant : 22 espèces en 2014, 43 en 2015 et 55 en 2016 amenant à un total de 74 espèces. Au total, 87 espèces d'abeilles sauvages ont été recensées jusqu'alors à Paris intramuros.

\section{Analyse des traits fonctionnels}

Parmi les 87 espèces capturées de 2011 à 2016, seulement 7 espèces sont des espèces coucous, soit $8 \%$ de la richesse spécifique et $1,6 \%$ de l'abondance. Parmi les 80 autres espèces, 12 sont oligolectiques : 6 espèces sont spécialisées pour les Asteracées ; 2 espèces pour les Fabacées ; 2 pour les Campanulacées ; 1 pour les Apiacées et une dernière pour les Borraginacées du genre Echium. Les 78 espèces restantes sont donc polylectiques dont 5 espèces possèdent tout de même des préférences pour certaines familles de plantes.

La plupart des espèces inventoriées sont solitaires $(88,7 \%), 8$ espèces $(9 \%)$ sont des bourdons au mode de vie eusocial à l'exception d'un entre eux, Bombus vestalis qui est un cleptoparasite et 2 espèces d'Halictidae sont sociales $(2,3 \%)$ : Halictus scabiosae et 

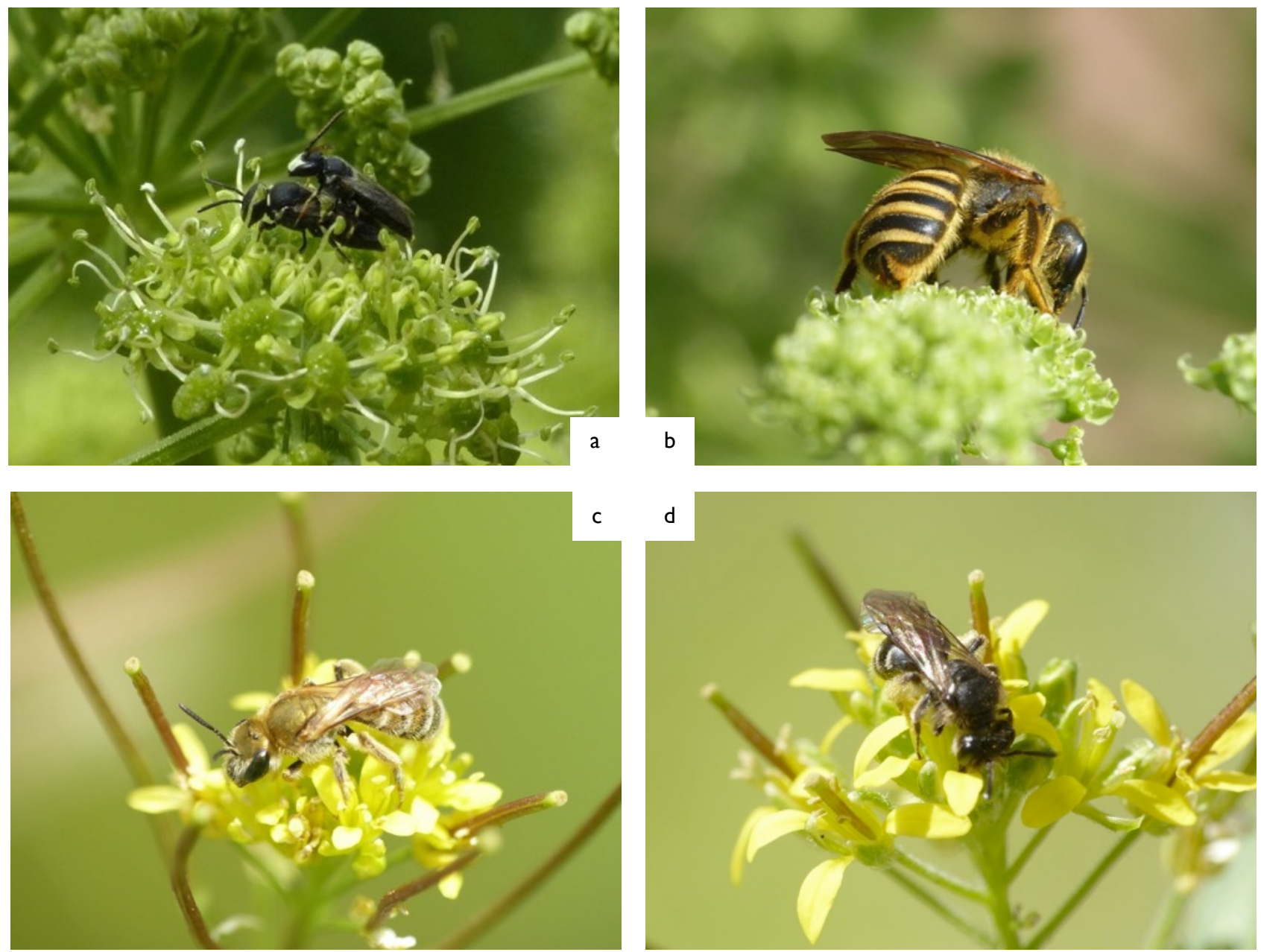

Figure $n^{\circ} 2$. (a) Couple d'Hylaeus sp.sur Angelica archangelica, (b) femelle d'Halictus scabiosae sur A. archangelica au Parc de Bercy en 2015,

(c) femelle de Seladonia sp. sur Sisymbrium irio et (d) femelle de Andrena sp. sur S. irio au Jardin des Plantes en 2015.

Lasioglossum malachurum. Sur les 80 espèces non cleptoparasites, 55 sont terricoles, 14 sont cavicoles, 8 sont rubicoles, 1 est xylocole (Xylocopa violacea). Enfin, 3 espèces peuvent nicher dans deux substrats différents.

Pour finir, seulement 3 espèces récoltées sont classées comme quasi-menacées sur la liste rouge européenne des abeilles sauvages (NIETO et al. 2014) : Lasioglossum pygmaeum, Lasioglossum sabulosum, et Lasioglossum sexnotatum. Toutes les autres espèces sont classées en préoccupation mineure (LC) ou les données qui les concernent sont insuffisantes pour statuer sur leur état de conservation (DD).

\section{Discussion}

Sur les 4 années au cours desquelles des inventaires ont été effectués dans Paris, la liste d'abeilles parisiennes totalise 87 espèces pour 1081 individus. Cela correspond à près de $9 \%$ du nombre d'espèces présentes en France, qui dépasse 970 espèces. L'ajout de la capture au filet a été bénéfique pour détecter la présence de 11 espèces supplémentaires ce qui témoigne de la nécessité de coupler les méthodes d'échantillonnages.

Avec au moins 87 espèces d'abeilles présentes à Paris, cette dernière se classe parmi les villes les plus riches en diversité derrière le Grand Lyon avec 291 espèces (ForTEL et al. 2014), Berlin avec 262 espèces (SAURE 1996), New York avec 110 espèces (FRETRIDGE et al. 2008) et enfin Poznan (Pologne) avec 104 espèces différentes (BANASZAK et al. 2011). Cette diversité existe en dépit de la faible superficie d'espaces verts dans la capitale française. En effet, les habitants de Berlin disposent de $31 \mathrm{~m}^{2}$ d'espaces verts, ceux de New York disposent de $23 \mathrm{~m}^{2}$ contre entre 5 et $14 \mathrm{~m}^{2}$ pour les habitants de Paris. Bien sûr, ces résultats doivent être interprétés avec beaucoup de prudence. Tout d'abord, il existe d'autres travaux portant sur les abeilles sauvages à Paris 
qui n'ont pas été compilés dans cet article et qui pourraient enrichir la liste d'espèces (FERRAND et al. 2014 ; StallegGer 2015 ; Plateaux 2017). De plus, les villes ne diffèrent pas seulement par leur mode d'occupation du sol mais également par leur climat, leur nombre d'habitants, leur superficie, et également par la nature de leur aire urbaine dominée ou non par des espaces agricoles. Par exemple, les disponibilités en ressources florales et en sites de nidification influencent fortement les communautés d'abeilles sauvages en ville (WRAY et al. 2014). Les espèces présentes à Paris sont majoritairement terricoles $(68,7 \%)$ mais sont proportionnellement moins représentatives que pour l'ensemble de la faune française $(80 \%$ d'espèces terricoles) ce qui suggère un effet délétère de la superficie de surfaces imperméables. Comme dans l'étude de GESLIN et al. (2016), Lasioglossum morio est l'espèce dominante sur les 4 années de suivi, représentant $20,7 \%$ du total des individus capturés, suivie de Lasioglossum nitidulum $(12,7 \%)$ et Lasioglossum laticeps $(10,4 \%)$. Nous constatons également une augmentation de la proportion d'espèces cleptoparasites par rapport aux précédentes études, cette dernière passant de $4,5 \%$ à $8 \%$ de la totalité des espèces capturées, ce qui reste encore faible par rapport aux $17 \%$ observées dans le Grand Lyon (FORTEL et al. 2014) bien que cette dernière étude présente des méthodes de captures différentes. Même si au fil des années la liste des espèces parisiennes d'abeilles sauvages s'étend, cet assemblage continue de présenter des caractéristiques d'une communauté appauvrie, tant par la dominance d'une seule espèce que par la faible proportion d'abeilles cleptoparasites.

A ce jour, il n'existe toujours pas de consensus dans la communauté scientifique sur la capacité des villes à abriter une diversité importante d'abeilles sauvages. Les espaces urbanisés semblent néanmoins être surtout propices aux espèces généralistes et ubiquistes. L'absence de consensus tient en partie au fait que pour la majorité de ces villes, les connaissances restent lacunaires. Cette étude apporte de nouvelles données pour Paris intramuros mais elle ne fait pas la synthèse de tous les travaux effectués sur la capitale française. Ceci doit être la prochaine étape dans l'amélioration de nos connaissances. Nous appelons donc ici à une mutualisation de tous ces travaux afin d'obtenir une liste plus exhaustive des abeilles parisiennes.

\section{Remerciements}

Un grand merci à David Genoud, Matthieu Aubert et Eric Dufrêne pour les déterminations à l'espèce de toutes les abeilles collectées chaque année.

\section{Bibliographie}

APUR 2004. Développer le végétal à Paris. Atelier Parisien d'Urbanisme 13.

Atlas Hymenoptera [En ligne]. Disponible à l'adresse http://www.atlashymenoptera.net (consulté le 15 février 2018).

BALDOCK K ET AL., 2015. Where Is the UK' $\mathrm{S}$ Pollinator Biodiversity? The Importance of Urban Areas for Flower-Visiting Insects. Proceedings of the Royal Society Biological Sciences 282 : 20142849.

BANASZAK-CIBICKA W, ŻMIHORSKI M, 2012. Wild bees along an urban gradient: winners and losers. Journal of Insect Conservation 16 : 331343.

Ferrand M, Garrin M, Mériguet B, GADOuM S, 2014. Réalisation d'un inventairediagnostic entomologique dans le cadre d'une démarche Oasis-nature (portée par Humanité \& Biodiversité) dans le parc de la Villette (75). Rapport final 2012-2014. Établissement public du parc et de la grande halle de la Villette (EPPGHV), Office pour les insectes et leur environnement (OPIE). $90 \mathrm{p}$.

FETRIDGE ED, Ascher JS, LANGELlotto GA, 2008. The bee fauna of residential gardens in a suburb of New York City (Hymenoptera: Apoidea). Annals of the Entomological Society of America. 101 : 1067-1077.

FlorAbeille [EN Ligne]. Disponible à l'adresse http://florabeilles.org [consulté le 15 février 2018].

FORTEL L ET AL., 2014. Decreasing Abundance, Increasing Diversity and Changing Structure of the Wild Bee Community (Hymenoptera: Anthophila) along an Urbanization Gradient. PLOS ONE 9(8).

GESLIN B ET AL., 2016. The Bee Fauna of Large Parks in Downtown Paris, France. Annales de la Société Entomologique de France 9271 (April).

Goulson D, Nicholls E, Botias C, AND Rotheray E, 2015. Bee Declines Driven by Combined Stress from Parasites, Pesticides, and Lack of Flowers. Science 347 (6229).

GENOUD D, 2017. Synthèse des connaissances sur les hyménoptères apoïdes - prélèvements 
2002 - 2009 et étude 2014. Travaux de la Massane. Tome 108.

HALl DM ET AL., 2016. The City as a Refuge for Insect Pollinators. Conservation Biology 31 (1) : 24-29. doi: 10.1111/cobi.1284.

INSEE. Population légales 2014. Commune de Paris (75056). Disponible à l'adresse https:// ww w.insee.fr/fr/statistiques/2534314? geo=COM-75056 [consulté le 15 février 2018].

NiEto A ET AL., 2014. European Red List of Bees. Publication Office of the European Union, Luxembourg. http://ec.europa.eu/environment/ nature/conservation/species/redlist/downloads/ European_bees.pdf

PotT SG ET AL., 2016. Safeguarding Pollinators and Their Values to Human Well-Being. Nature 540 : 220-229. http://dx.doi.org/10.1038/ nature20588.

Ropars L, DAJOz I, Geslin B, 2017. La ville un désert pour les abeilles sauvages ? Journal de Botanique 79 : 29-35.

Roulston H, Smith SA, Brewster AL, 2007. A comparison of pan trap and intensive net sampling techniques for documenting a bee (Hymenoptera: Apiformes) Fauna. Journal of Kansas Entomological Society 80(2) : 179-181.

SAURE C, 1996. Urban habitats for bees : the example of city of Berlin. Dans The conservation of bees (eds Matheson A, Buchmann SL, O'toole C, Westrich $\mathrm{P}$ and Williams IH), pp. 47-52. London Academic Press.

STALLEGGER P, 2015. Inventaire et analyse du peuplement d'abeilles sauvages (Hymenoptera Apoidea Anthophila) de la Petite Ceinture ferroviaire de Paris. Dans Institut d'Ecologie Appliquée \& SETEC Environnement 2015. Rapport d'étude pour la Ville de Paris, Direction des Espaces Verts et de l'Environnement. 171 p.

WESTRICH P. Habitat requirements of central European bees and the problems of partial habitats. Dans The conservation of bees (eds Matheson A, Buchmann SL, O'toole C, Westrich $\mathrm{P}$ and Williams IH), pp. 1-16. London Academic Press.

WestPhal C ET AL., 2008. Measuring Bee Diversity in Different European Habitats and Biogeographical Regions. Ecological Monographs 78(4) : 653-71.

Wilson JS, Griswold T, \& Messinger, OJ, 2008 . Sampling Bee Communities (Hymenoptera: Apiformes) in a Desert Landscape : Are Pan Traps Sufficient? Journal of Kansas Entomological Society 81(3), 288-300.

Wray JC, NeAme LA, Elle E, 2014. Floral resources, body size, and surrounding landscape influence bee community assemblages in oaksavannah fragments. Ecological Entomology 39 : 83-93.

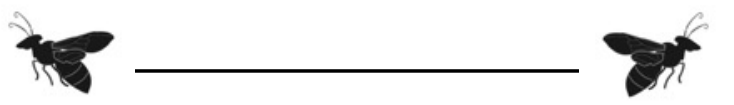

